\title{
Image Compression Based On Multiple Parameter Discrete Fractional Fourier Transform for Satellite and Medical Images
}

\author{
Deepak Sharma ${ }^{1}$, Rajiv Saxena ${ }^{2}$ and Narendra Singh $^{3}$ \\ Department of Electronics and Communication Engineering \\ 1,3 Jaypee University of Engineering and Technology, Guna, India \\ 2 Jaypee University, Anoopshahr, India \\ ${ }^{1}$ deepakforu23@rediffmail.com, ${ }^{2}$ rajiv.saxena@jiit.ac.in \\ ${ }^{3}$ narendra.singh@juet.ac.in
}

\begin{abstract}
With the growing demand of high quality multimedia $(H D)$ the data size has increased thus the compression is the essential requirement to process and store data with smaller size. The Multiple Parameter Discrete Fractional Fourier Transform (MPDFRFT) is generalization of the discrete fractional Fourier Transform and can be use for compression of high resolution images with the extra degree of freedom provided by the MPDFRFT and its different fractional orders finally decompressed image can also be recovered. This paper deals with the image compression based on MPDFRFT using Eigen vector decomposition algorithm. The MPDFRFT possesses all the desired properties of discrete fractional Fourier transform. The MPDFRFT converts to the DFRFT when all of its order parameters are the same. We exploit the properties of multiple-parameter DFRFT and propose a novel compression scheme for satellite and medical images more conveniently than urban, rural and natural images. In this scheme image is subdivided and MPDFRFT is applied for the subdivided image to form transformed coefficients and Inverse MPDFRFT is applied for reconstruction of original images. The proposed compression scheme with MPDFRFT significantly shows better results over fractional cosine transform (FRCT), Fourier transforms (FT) and cosine transforms $(C T)$. A comparison has been made between these techniques and observed that a good fidelity of decompressed image can be achieved at different fractional order parameter values of the transforms. The performance of system analyzed based on parameters like Peak Signal-to-Noise Ratio (PSNR), mean square error (MSE) and Compression Ratio (CR). The MPDFRFT provides better mean square error (MSE) and peak signal noise ratio (PSNR) for the same compression ratio (CR) as compared to FRCT, FT, cosine transform and classical lifting scheme based on wavelet, during image processing using MATLAB platform.
\end{abstract}

Keywords: Satellite Image Compression, Medical Image Compression, Discrete Fractional Fourier Transform (DFRFT), Fourier Transform (FT), Fractional Fourier Transform (FRFT), Multiple Parameter Discrete Fractional Fourier Transform (MPDFRFT), PSNR, MSE

\section{Introduction}

Image and video data compression refers to a process in which the amount of data used to represent image and video is reduced to meet a bit rate requirement while the quality of the reconstructed image or video satisfies a requirement for a certain application and the complexity of computation involved is affordable for the application. It is a process intended to yield a compact representation of an image, 
thereby reducing the image storage/transmission requirements. Its feasibility rests with two types of redundancies, i.e., statistical redundancy and psychovisual redundancy [1]. Statistical redundancy can be classified into two types: interpixel redundancy and coding redundancy. By interpixel redundancy we mean that pixels of an image frame and pixels of a group of successive image or video frames are not statistically independent. On the contrary, they are correlated to various degrees. This type of interpixel correlation is referred to as interpixel redundancy. Interpixel redundancy can be divided into two categories, spatial redundancy and temporal redundancy. Spatial redundancy represents the statistical correlation between pixels within an image frame. Hence it is also called intraframe redundancy. Temporal redundancy is concerned with the statistical correlation between pixels from successive frames in a temporal image or video sequence. Therefore, it is also called interframe redundancy. By coding redundancy we mean the statistical redundancy associated with coding techniques. Psychovisual redundancy originates from the characteristics of the human visual system (HVS). It is due to data that is ignored by the human visual system. Compression techniques reduce the number of bits required to represent an image by taking advantage of these redundancies. The objective of compression is to reduce the number of bits as much as possible, while keeping the resolution and the visual quality of the reconstructed image as close to the original image as possible.

The image compression techniques are broadly classified into two categories Lossless compression and Lossy compression technique [2]. In lossless compression techniques, the original image can be perfectly recovered from the compressed (encoded) image. These are also called noiseless since they do not add noise to the signal (image). It is also known as entropy coding since it use statistics/decomposition techniques to eliminate/minimize redundancy. Lossless compression is used only for a few applications with stringent requirements such as medical imaging.

Following techniques are included in lossless compression:

\section{$\begin{array}{llll}\text { 1. Run length encoding } & \text { 2. Huffman encoding } & \text { 3. LZW coding } \quad \text { 4. Area coding }\end{array}$}

Lossy schemes provide much higher compression ratios than lossless schemes. Lossy schemes are widely used since the quality of the reconstructed images is adequate for most applications. By this scheme, the decompressed image is not identical to the original image, but reasonably close to it. In lossy compression techniquestransformation - decomposition process is completely reversible . The quantization process results in loss of information. The entropy coding after the quantization step, however, is lossless. The decoding is a reverse process. Firstly, entropy decoding is applied to compressed data to get the quantized data. Secondly, dequantization is applied to it and finally the inverse transformation to get the reconstructed image. Lossy compression techniques includes following schemes:

$\begin{array}{llll}\text { 1. Transformation coding } & \text { 2. Vector quantization } & 3 \text {. Fractal coding } & 4 \text {. Block }\end{array}$ Truncation Coding 5. Subband coding.

All the contemporary image compression schemes use various mathematical transforms for compression. The compression performance is closely related to the performance by these mathematical transforms in terms of energy compaction and spatial frequency isolation by exploiting inter-pixel redundancies present in the image data. Transform coding constitutes an integral component of contemporary image/video processing applications. Transform coding relies on the premise that pixels in an image exhibit a certain level of correlation with their neighboring pixels. Similarly in a video 
transmission system, adjacent pixels in consecutive frames show very high correlation. Consequently, these correlations can be exploited to predict the value of a pixel from its respective neighbors. A transformation is, therefore, defined to map this spatial (correlated) data into transformed (uncorrelated) coefficients. Clearly, the transformation should utilize the fact that the information content of an individual pixel is relatively small.

In much extracted manner, the fractional Fourier transform (FRFT) is a generalization of the ordinary Fourier transform in time and frequency plane for the analysis and processing of non stationary or time varying signals [3]. The idea of fractional Fourier operator much established in 1980 with publications by Namias [4]. Santhanam et al., [5] defined a discrete fractional Fourier transform (DFrFT) by using Taylor series expansion of the DFT matrix followed by the application of CayleyHamilton theorem and defined DFrFT as a weighted sum of its powers. DFrFT can mainly be split in to two major groups. The first approach is based on the $\mathrm{S}$ matrix introduced by Dickinson et al., [6]. Second approach Candan et al., used the secondorder difference equation by approximating the second-order differential equation in which the homogenous solution set is the Hermite-Gauss functions [7]. Later on Candan uses higher-order approximations to discrete derivative operator to approximate Hermite-Gauss functions [8]

In Pei and Yeh defined the DFRFT based on the eigen decomposition of the DFT matrix, a DFRFT with one fractional parameter was defined by taking fractional eigen value powers of an eigen decomposition of the DFT matrix [9]. The DFT eigenvectors used in are Hermite -Gaussian function type [9]. These eigenvectors are computed from a DFT-commuting matrix proposed in [6] by Dickson and Steigletz. Pei et al. [9], first proposed the eigen decomposition- based definition of the DFRFT and then Candan et al.[7] consolidated this definition. Hanna et al. considered generation eigenvectors by the singular value decomposition method and direct batch evaluation [10-12]. It finds application in signal image processing due to high decorrelation among the coefficients and compact signal representation in FRFT domain [3, 4, 13].

The research article is organized as in a following way: In preliminaries section 2 the FRFT, DFRFT and MPDFRFT are discussed with their mathematical definition and properties. In subsection 2.3 the way to deduce MPDFRFT from DFRFT along with their algorithm in mathematical steps are illustrated. In section 3 the major focus is on the proposed scheme for image compression based on multiple parameters discrete fractional Fourier transform (MPDFRFT). The segment discusses each block implementation method and their conceptual significance. The section 4 highlights the major performance measuring parameters in compression domain. The section 5 is solely dedicated to simulation results and discussion. In this segment also the comparison is done with similar earlier existed schemes. In section 6 the research article is concluded on the basis of comparison in a summarized way.

\section{Preliminaries}

\subsection{Fractional Fourier Transform}

The FRFT of the signal $x(t)$ by angle $\alpha$ is expressed as,

$$
f_{\alpha}(u)=\int_{-\infty}^{+\infty} x(t) K_{\alpha}(t, u) d t
$$

The signal $x(t)$ can be recovered back by an FRFT with an angle $-\alpha$ : 


$$
x(t)=\int_{-\infty}^{+\infty} f_{\alpha}(u) K_{-\alpha}(t, u) d u
$$

The FRFT transform kernel is given by $[4,13]$

$$
K_{\alpha}(t, u)= \begin{cases}\sqrt{\frac{1-j \cot \alpha}{2 \pi}} e^{j\left(t^{2}+u^{2}\right) / 2 \cot \alpha-j u t \operatorname{cosec} \alpha} & \text { if } \alpha \text { is not a multipleof } \pi \\ \delta(t-u) & \text { if } \alpha \text { is not a multipleof } 2 \pi \\ \delta(t+u) & \text { if } \alpha+\pi \text { is not a multipleof } 2 \pi\end{cases}
$$

Where $K_{\alpha}(t, u)=\sum_{n=0}^{\infty} e^{-j o n} H_{n}(t) H_{n}(u)$ and $H_{n}(t)=\frac{1}{\sqrt{2^{n} n ! \sqrt{\pi}}} h_{n}(t) e^{-t^{2} / 2}$

Where $\alpha$ indicates the rotation angle of the transformed signal for FRFT. $H_{n}(t)$ is the $n$-th order normalized Hermite Gaussian function. Where $h_{n}(t)$ is the $n$-th order Hermite polynomial.

The 2D FRFT of the signal $x(s, t)$ by angle parameter $(\alpha, \beta)$ is given by [14],

$$
f_{\alpha, \beta}(u, v)=\int_{-\infty}^{+\infty} x(s, t) K_{\alpha, \beta}(s, t, u, v) d s d t
$$

The signal $x(s, t)$ can be recovered by a 2D FRFT operation with angles $-\alpha,-\beta$ :

$$
x(s, t)=\int_{-\infty}^{+\infty} f_{\alpha, \beta}(u, v) K_{-\alpha,-\beta}(u, v, s, t) d u d v
$$

The 2D FRFT transform kernel with various orders in two dimensions is defined as [14],

$K_{\alpha, \beta}(s, t, u, v)=\frac{1}{2 \pi} \sqrt{1-j \cot \alpha} \sqrt{1-j \cot \beta} e^{\left[j\left(s^{2}+u^{2}\right) / 2 \cot \alpha-j u s \operatorname{cosec} \alpha\right]} e^{\left[j\left(t^{2}+v^{2}\right) / 2 \cot \beta-j t v \operatorname{cosec} \beta\right]}$

Where $\alpha$ and $\beta$ indicate the rotation angles of the transformed signal for 2D FRFT.

\subsection{Discrete Fractional Fourier Transform}

The $a$-th order $N \times N$ DFRFT is developed based on the eigen decomposition, and its transform kernel is given on the basis of $[7,9,15]$ is,

$$
F^{2 \alpha / \pi}=V D^{2 \alpha / \pi} V^{T}
$$

Here $a=2 \alpha / \pi$ the DFRFT order of the parameter and $\alpha$ indicates the rotation angle of DFRFT.B.W. Dickinson and K. Steiglitz [6] introduced a commuting matrix $S$ to compute the real eigenvectors of the DFT kernel matrix $\boldsymbol{F}$.

$$
S=\left[\begin{array}{cccccc}
2 & 1 & 0 & 0 & \vdots & 1 \\
1 & 2 \operatorname{Cos} \omega & 1 & 0 & \vdots & 0 \\
0 & 1 & 2 \operatorname{Cos} 2 \omega & 1 & \vdots & 0 \\
\vdots & \vdots & \vdots & 2 \operatorname{Cos} 3 \omega & \vdots & \vdots \\
\vdots & \vdots & \vdots & \vdots & \vdots & \vdots \\
1 & 0 & 0 & 0 & \vdots & 2 \operatorname{Cos}(N-1) \omega
\end{array}\right]
$$


Where, $\omega=2 \pi / N$ and $N$ is the size of the DFT kernel matrix. Matrix $S$ commutes with matrix $\boldsymbol{F}$, The eigenvectors of matrix $\boldsymbol{S}$ are also the eigenvectors of matrix $\boldsymbol{F}$, but their eigen values are distinct. Because matrix $S$ is a real symmetric matrix, the eigen values of $S$ are all real and the eigenvectors of $\boldsymbol{S}$ are orthonormal to each other.

$\mathrm{V}=\left[\mathrm{v}_{0}\left|\mathrm{v}_{1}\right| \ldots \ldots .\left|\mathrm{v}_{\mathrm{N}-2}\right|\left|\mathrm{v}_{\mathrm{N}-1}\right|\right]$ For $N$ is odd, $\mathrm{V}=\left[\mathrm{v}_{0}\left|\mathrm{v}_{1}\right| \ldots \ldots .\left|\mathrm{v}_{\mathrm{N}-2}\right|\left|\mathrm{v}_{\mathrm{N}-1}\right|\right]$ for $\mathrm{N}$ is even, and $v_{k}$ is the $k$-th order DFT hermit eigen vector. $D^{2 \alpha / \pi}$ is a diagonal matrix with eigen values of DFRFT in the diagonal entries. The methods for finding the DFT Hermite eigenvectors $v_{k}$ are presented in [9] and [15]. In Table 1, there exists a jump in the last eigen values for the two even-length cases.

The $N \times N$ DFT matrix $F$ is given by,

$$
F_{k n}=\frac{1}{\sqrt{N}} e^{-j \frac{2 \pi}{N} k n} \quad 0 \leq k, n \leq N-1
$$

\section{Table 1. The Eigen Value Assignment for DFRFT Kernel}

\begin{tabular}{|c|c|c|}
\hline No. & $N$ & Eigen Values \\
\hline 1. & $4 m$ & $e^{-j k \alpha}, k=0,1,2 \ldots .,(4 m-2), 4 m$ \\
\hline 2. & $4 m+1$ & $e^{-j k \alpha}, k=0,1,2 \ldots,(4 m-1), 4 m$ \\
\hline 3. & $4 m+2$ & $e^{-j k \alpha}, k=0,1,2 \ldots, 4 m,(4 m+2)$ \\
\hline 4. & $4 m+3$ & $e^{-j k \alpha} k=0,1,2 \ldots,(4 m+1),(4 m+2)$ \\
\hline
\end{tabular}

Therefore, there are some differences in computing the DFRFT kernels between even- and odd-length cases. For the odd- and even- length cases, equation (1) can be written as follows:

$$
\begin{gathered}
F^{2 \alpha / \pi}=\sum_{k=0}^{N-1} e^{-j k \alpha} v_{k} v_{k}^{T} \quad(\text { For the odd values of } \mathrm{N}) \\
\left.F^{2 \alpha / \pi}=\sum_{k=0}^{N-2} e^{-j k \alpha} v_{k} v_{k}^{T}+e^{-j N \alpha} v_{N} v_{N}^{T} \quad \text { (For the even values of } \mathrm{N}\right)
\end{gathered}
$$

The DFRFT output is computed as a,

$$
\begin{gathered}
X_{\alpha}=\sum_{k=0}^{N-1} e^{-j k \alpha} v_{k} v_{k}^{T} x \quad(\text { For the odd values of } \mathrm{N}) \\
X_{\alpha}=\sum_{k=0}^{N-2} e^{-j k \alpha} v_{k} v_{k}^{T} x+e^{-j N \alpha} v_{N} v_{N}^{T} x
\end{gathered}
$$

\subsection{Multiple Parameter Discrete Fractional Fourier Transform}

The $a$-th order DFRFT matrix is $F^{2 \alpha / \pi}$ given in equation (7). We know that $F^{2 \alpha / \pi}$ degenerates to the DFT matrix $F$ in equation (7) when $a=1$. So the DFRFT is a generalization of the DFT. If we further generalize the DFRFT on the basis of taking different fractional power for the eigen values $\lambda_{k}=\exp (-j \pi k / 2)$ of the DFT matrix. Subsequently the $N$ point $N \times N$ MPDFRFT matrix is,

$$
F^{\overline{2 \alpha / \pi}}=V \cdot \operatorname{diag}\left(\left(e^{-j \frac{\pi}{2} 0}\right)^{a_{0}},\left(e^{-j \frac{\pi}{2} 1}\right)^{a_{1}} \ldots \ldots \ldots \ldots . .\left(e^{-j \frac{\pi}{2}(N-1)}\right)^{a_{N-1}}\right) \cdot V^{T} \text { Odd values of } \mathrm{N}
$$




$$
=V \cdot \operatorname{diag}\left(\left(e^{-j \frac{\pi}{2} 0}\right)^{a_{0}},\left(e^{-j \frac{\pi}{2} 1}\right)^{a_{1}} \ldots \ldots .\left(e^{-j \frac{\pi}{2}(N-2)}\right)^{a_{N-2}}\left(e^{-j \frac{\pi}{2}(N)}\right)^{a_{N}}\right) \cdot V^{T} \text { Even values of } \mathrm{N}(15)
$$

When $\operatorname{diag}\left(u_{1}, u_{2}, \ldots \ldots, u_{n}\right)$ represents the $N \times N$ diagonal matrix whose diagonal elements are $u_{1}, u_{2}, \ldots \ldots, u_{n}$. In equation (15), $\bar{a}$ is a $1 \times N$ parameter vector consisting of the $N$ independent order parameters of the MPDFRFT,

$$
\bar{a}= \begin{cases}\left(a_{0}, a_{1}, a_{2}, \ldots \ldots . ., a_{N-1}\right) & \text { for } \mathrm{N} \text { odd } \\ \left(a_{0}, a_{1}, a_{2}, \ldots \ldots . ., a_{N-2}, a_{N}\right) & \text { for } \mathrm{N} \text { even }\end{cases}
$$

The diagonal matrix is simplified as

$$
D^{\overline{2 \alpha / \pi}}=\left\{\begin{array}{l}
\operatorname{diag}\left(\left(e^{-j \frac{\pi}{2} 0}\right)^{a_{0}},\left(e^{-j \frac{\pi}{2} 1}\right)^{a_{1}} \ldots \ldots \ldots \ldots . . .\left(e^{-j \frac{\pi}{2}(N-1)}\right)^{a_{N-1}}\right) \text { for } \mathrm{N} \text { is odd } \\
\operatorname{diag}\left(\left(e^{-j \frac{\pi}{2} 0}\right)^{a_{0}},\left(e^{-j \frac{\pi}{2} 1}\right)^{a_{1}} \ldots \ldots\left(e^{-j \frac{\pi}{2}(N-2)}\right)^{a_{N-2}}\left(e^{-j \frac{\pi}{2}(N)}\right)^{a_{N}}\right) \text { For } \mathrm{N} \text { even }
\end{array}\right.
$$

The vector $\bar{a}$ is given in equation (16) and $D^{\overline{2 \alpha / \pi}}$ is the $N \times N$ diagonal matrix of the DFT Eigen values

$$
D^{2 \alpha / \pi}= \begin{cases}\operatorname{diag}\left(e^{-j \frac{\pi}{2} 0}, e^{-j \frac{\pi}{2} 1} \ldots \ldots \ldots . . e^{-j \frac{\pi}{2}(N-1)}\right) & \text { for N is odd } \\ \operatorname{diag}\left(e^{-j \frac{\pi}{2} 0}, e^{-j \frac{\pi}{2} 1} \ldots \ldots \ldots . . e^{-j \frac{\pi}{2}(N-2)}, e^{-j \frac{\pi}{2}(N)}\right) & \text { For N even }\end{cases}
$$

Then equation (13) can be expressed in summarized form as,

$$
F^{\overline{2 \alpha / \pi}}=V D^{\overline{2 \alpha / \pi}} V^{T}
$$

The MPDFRFT of $X_{\bar{a}}$ of the $N \times 1$ data vector $\mathrm{x}$ with the parameter vector $\bar{a}$ can be given by,

$$
X_{\bar{a}}=F^{\overline{2 \alpha / \pi}} \mathrm{x}
$$

The main features of the MPDFRFT are discussed as follows.

1. If $\bar{a}=(a, a, \ldots ., a)$, the MPDFRFT is converted into DFRFT so DFRFT is the special condition of the MPDFRFT.

2. The $N$-point MPDFRFT can have up to $\mathrm{N}$ independent and possibly different order parameters, Whereas DFRFT have only one order parameter.

3. The computation complexity for the MPDFRFT is $O\left(N^{2}\right)$ same as DFRFT. 
Table 2. Properties of MPDFRFT

\begin{tabular}{|c|c|c|}
\hline No. & Property & \multicolumn{1}{|c|}{ Mathematical representation } \\
\hline 1. & Unitarity & $\left(F^{\overline{2 \alpha / \pi}}\right)^{H}\left(F^{\overline{2 \alpha / \pi}}\right)=\left(V D^{\overline{2 \alpha / \pi}} V^{T}\right)^{H}\left(V D^{\overline{2 \alpha / \pi}} V^{T}\right)=V^{T}=I$ \\
\hline 2. & Identity Matrix & $\bar{a}=\overline{0}=(0,0, \ldots, 0), F^{\overline{2 \alpha / \pi}}=V D^{\overline{0}} V^{T}=V V^{T}=I$ \\
\hline 3. & Fourier Transform (F) & $\bar{a}=\overline{1}=(1,1, \ldots, 1), F^{\overline{2 \alpha / \pi}}=V D^{\overline{1}} V^{T}=V D V^{T}=F$ \\
\hline 4. & Index additivity & $\left(F^{\overline{2 \alpha_{1} / \pi}}\right)\left(F^{\overline{2 \alpha_{2} / \pi}}\right)=\left(V D^{\bar{a}_{1}} V^{T}\right)\left(V D^{\bar{a}_{2}} V^{T}\right)=\left(V D^{\bar{a}_{1}+\bar{a}_{2}} V^{T}\right)=F^{\overline{2\left(\bar{\alpha}_{1}+\bar{\alpha}_{2}\right) / 2}}$ \\
\hline 5. & Index Commutativity & $\left(F^{\overline{2 \alpha_{1} / \pi}}\right)\left(F^{\overline{2 \alpha_{2} / \pi}}\right)=\left(V D^{\bar{a}_{1}+\bar{a}_{2}} V^{T}\right)=V D^{\bar{a}_{2}+\bar{a}_{1}} V^{T}=\left(F^{\overline{2 \alpha_{2} / \pi}}\right)\left(F^{\overline{2 \alpha_{1} / \pi}}\right)$ \\
\hline 6. & Inverse Transform & $\left(F^{\overline{2 \alpha / \pi})^{-1}}=\left(F^{-\overline{2 \alpha / \pi}}\right)\right.$ \\
\hline 7. & Periodicity & $e^{-j \frac{\pi}{2} k\left(a_{k}+\frac{4}{k}\right)}=e^{-j \frac{\pi}{2} k a_{k}}$, if $k \neq 0$ and $\left(e^{-j \frac{\pi}{2} 0}\right)^{a_{0}}=1$ \\
\hline
\end{tabular}

\section{Proposed Model for Image Compression and Decompression}

Earlier The Frct and FRFT based image compression schemes are established by the researcher here in Figure 1. The compression scheme is proposed on the basis of multiple parameter discrete fractional Fourier transform with few additional blocks are augmented and implemented to improve the performance of the proposed model.

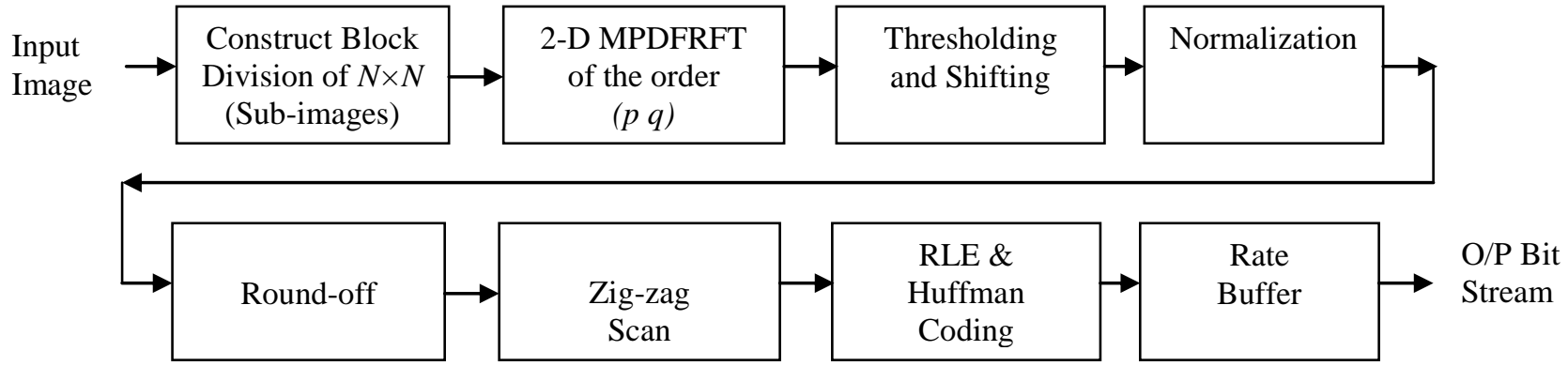

Figure 1. Image Compression Model based on MPDFRFT

Similarly at the receiver side the reverse process of decompression is applied with decoding and 2D MPDFRFT is applied with its reverse order subsequently the block merger is applied as shown in Figure 2 below.

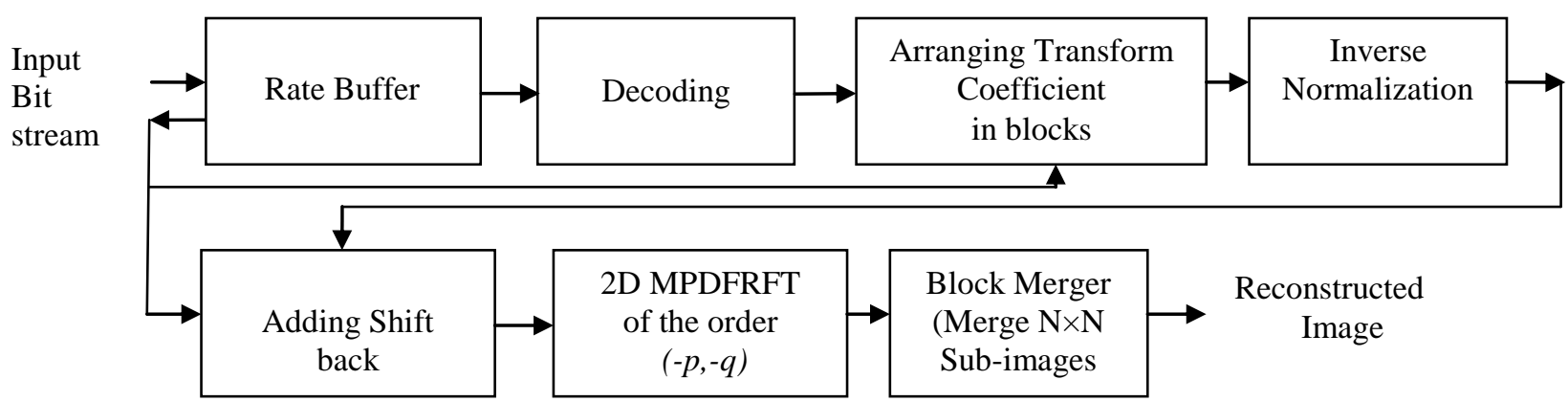

Figure 2. Image Decompression Model based on MPDFRFT 
Transform coding based on utilization of interpixel correlation. Transform coding has been found to be efficient in coding prediction error in motion compensated predictive coding. We summarize the procedures of transform coding. There are three major steps in transform coding block division, linear transform and bit allocation. The selection of subimage (block) size, $N$, is important. Normally, the larger the size the more uncorrelation in transform coding can achieve. However, that the correlation between image pixels becomes insignificant when the distance between pixels becomes large. On the other hand, a large size causes a possibly severe effect of transmission error on reconstructed images problems. 2D MPDFRFT transform is used in image compression is symmetric (hence separable) and unitary, the matrix form of the $2-\mathrm{D}$ image transform can be expressed as $T=F^{T} G F$.

For an image "L" of $R \times S$, is converted into $N \times N$ subimages L' preferred $8 \times 8$ image size and then applied 2D-MPDFRFT to L' size with parameters vectors $(p, q)$ is given by

$$
L_{(p, q)}^{\prime}=F^{p} \cdot L^{\prime} \cdot F^{q}
$$

Where $F^{p}$ and.$F^{q}$ are the 256 point MPDFRFT matrices respectively, $p$ and $q$ are the parameter vectors of sizes $1 \times 256$ and $1 \times 256$ matrices respectively. Here $p$ and $q$ are the parameter vectors distributed over the interval $[0,4]$. The variances of these transform coefficients, and therefore the signal energy associated with the transform coefficients, can be arranged in a ascending order. It can be shown that the total energy before and after the transform remains the same. Therefore, the more energy compacted in a fraction of total coefficients, the better energy compaction the transform has. The transformed blocks go through truncation, quantization, and codeword assignment. The last three functions: truncation, quantization, and codeword assignment, are combined and called bit allocation. It is known that the applied transform decorrelates subimages. Moreover, it redistributes image energy in the transform domain in such a way that most of the energy is compacted into a small fraction of coefficients. Therefore, it is possible to discard the majority of transform coefficients without introducing significant distortion. We see that in transform coding there are mainly three types of errors involved. One is due to truncation. That is, the majority of coefficients are truncated to zero. Others come from quantization. There are two different ways to truncate transform coefficients. One is called zonal coding, while the other is threshold coding. In threshold coding, also known as threshold sampling, there is not a predefined zone [16]. Instead, each transform coefficient is compared with a threshold. If it is smaller than the threshold, then it is set to zero. If it is larger than the threshold, it will be retained for quantization and encoding. Compared with zonal coding, this scheme is adaptive in truncation in the sense that the coefficients with more energy are retained no matter where they are located. The addresses of these retained coefficients, however, have to be sent to the receiver as side information. Furthermore, the threshold is determined after an evaluation of all coefficients. Hence, it was usually a two-pass adaptive technique. In thresholding and Shifting we use $C(u, v)$ to denote the MPDFRFT coefficients. The DC coefficient, $C(0,0)$ is processed differently, the DC coefficients are encoded with a differential coding technique. For all the AC coefficients, the following thresholding and shifting are,

$$
C_{T}(u, v)=\left\{\begin{array}{lll}
C(u, v)-T & \text { if } & C(u, v)>T \\
0 & \text { if } & C(u, v) \leq T
\end{array}\right.
$$


Where, $T$ on the right-hand side is threshold. Note that the above equation also implies a shifting of transform coefficients by $T$ when $C(u, v)>T$. The threshold value is adjusted by the feedback from the rate buffer, or by the desired bit rate.

The threshold subtracted transform coefficients $C_{T}(u, v)$ are normalized before round off. The normalization is as follows:

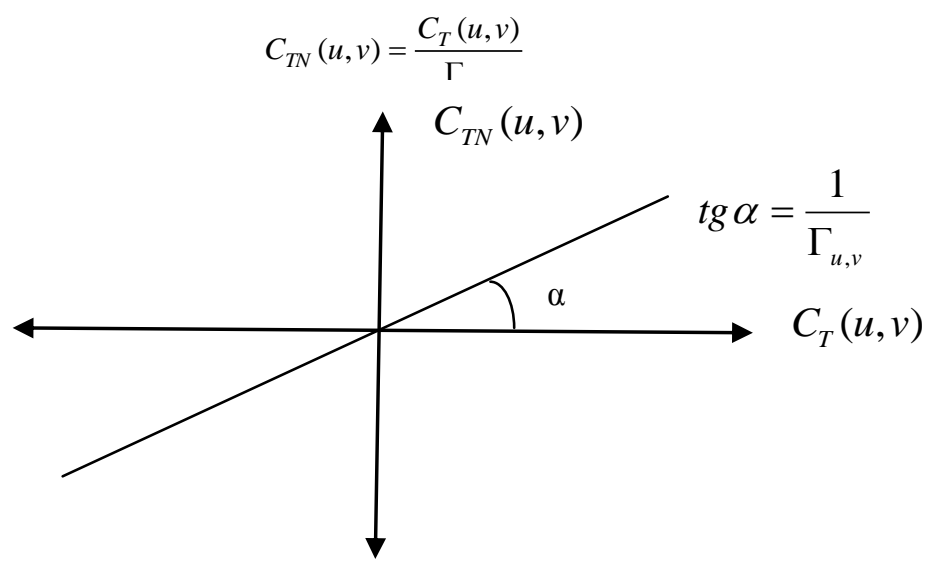

\section{Figurr 3. (a) Input-output Characteristic of Normalization}

Where the normalization factor $\Gamma_{u, v}$ is controlled by the rate buffer. The round off process converts floating point to integer as follows.

$$
R\left[C_{T N}(u, v)\right]=C_{T N}(u, v)= \begin{cases}C_{T N}(u, v)+0.5 & \text { if } C_{T N}(u, v) \geq 0 \\ C_{T N}(u, v)-0.5 & \text { if } C_{T N}(u, v)<0\end{cases}
$$

The combination of normalization and round off is equivalent to a uniform midtread quantizer with the quantization step size equal to the normalization factor $\Gamma_{u, v}$. Normalization is a scaling process, which makes the resultant uniform midtread quantizer adapt to the dynamic range of the associated transform coefficient.

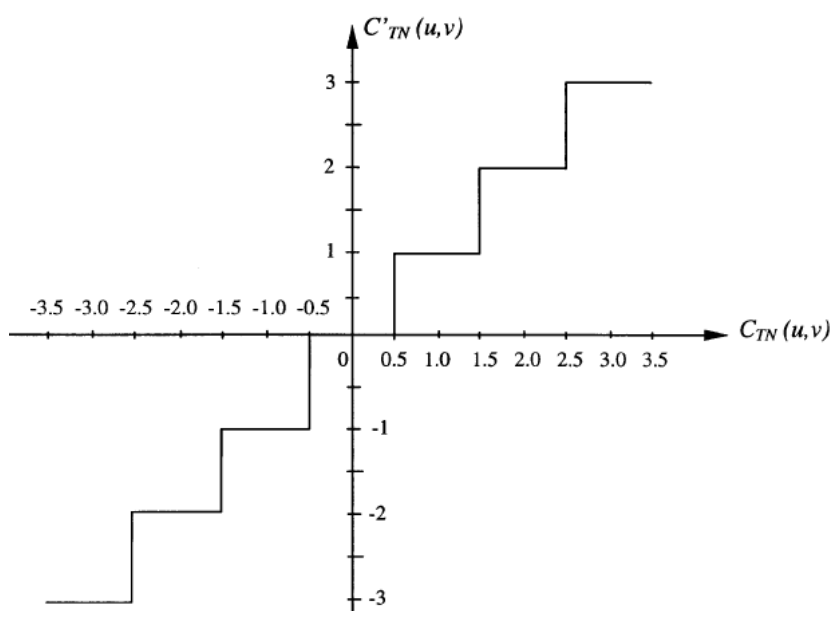

Figure 3. (b) Input-output characteristic of Round Off 
A matrix consisting of all the normalization factors is called a quantization table 3 in JPEG. A given luminance quantization table 3(a) and a Chrominance quantization table 3(b) are used for JPEG.

Table 3. Quantization Table

\section{(a) Luminance Quantization Table}

\begin{tabular}{|c|c|c|c|c|c|c|c|}
\hline 16 & 11 & 10 & 16 & 24 & 40 & 51 & 61 \\
\hline 12 & 12 & 14 & 19 & 26 & 58 & 60 & 55 \\
\hline 14 & 13 & 16 & 24 & 40 & 57 & 69 & 56 \\
\hline 14 & 17 & 22 & 29 & 51 & 87 & 80 & 62 \\
\hline 18 & 22 & 37 & 56 & 68 & 109 & 103 & 77 \\
\hline 24 & 35 & 55 & 64 & 81 & 104 & 113 & 92 \\
\hline 49 & 64 & 78 & 87 & 103 & 121 & 120 & 101 \\
\hline 72 & 92 & 95 & 98 & 112 & 100 & 103 & 99 \\
\hline
\end{tabular}

\section{(b) Chrominance Quantization Table}

\begin{tabular}{|l|l|l|l|l|l|l|l|}
\hline 17 & 18 & 24 & 47 & 99 & 99 & 99 & 99 \\
\hline 18 & 21 & 26 & 66 & 99 & 99 & 99 & 99 \\
\hline 24 & 26 & 56 & 99 & 99 & 99 & 99 & 99 \\
\hline 47 & 66 & 99 & 99 & 99 & 99 & 99 & 99 \\
\hline 99 & 99 & 99 & 99 & 99 & 99 & 99 & 99 \\
\hline 99 & 99 & 99 & 99 & 99 & 99 & 99 & 99 \\
\hline 99 & 99 & 99 & 99 & 99 & 99 & 99 & 99 \\
\hline 99 & 99 & 99 & 99 & 99 & 99 & 99 & 99 \\
\hline
\end{tabular}

Zigzag scanning minimizes the use of run-length codes in the block given by chen and Pratt in [16]. The application of the Huffman coding to the magnitude of nonzero transform coefficients and run-lengths coding for zero transforms. A rate buffer accepts a variable-rate data input from the encoding process and provides a fixed-rate data output to the channel. The status of the rate buffer is monitored and fed back to control the threshold and the normalization factor. The threshold and the normalization factor are controlled by rate buffer feedback. Since the threshold decides how many transform coefficients are retained and the normalization factor is actually the quantization step size, the rate buffer has direct impact on the bit rate of the transform coding system. After thresholding, normalization and roundoff are applied to the transform coefficients in a block; a great majority of transform coefficients are set to zero. A zigzag scan can convert the 2-D array of transform coefficients into a 1-D sequence as shown in figure 4 below. The number of consecutive zero-valued coefficients in the 1-D sequence is referred to as the run-length of zeros and is used to provide address information of nonzero transformed coefficients. Both the magnitude of nonzero coefficients and run-length information need to be coded.

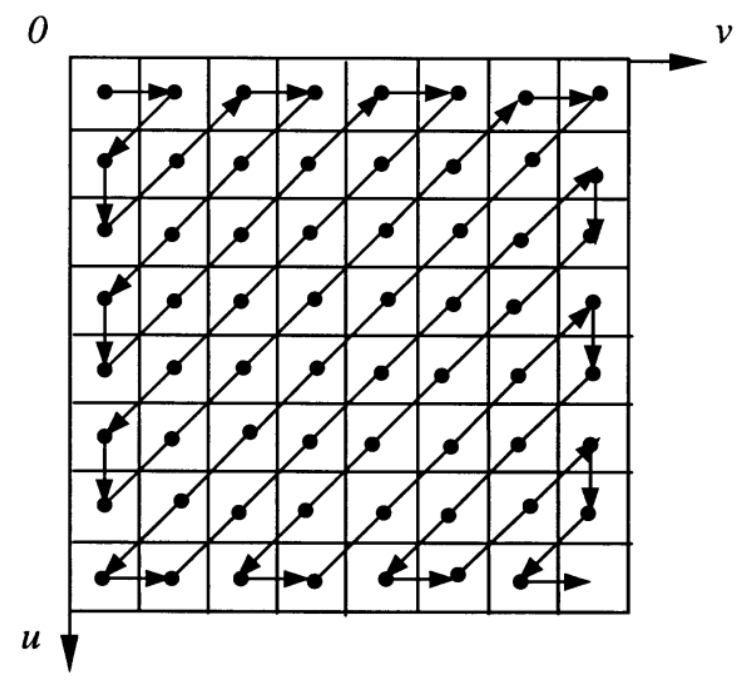

Figure 4. Zig-zag Scanning of Coefficient within a $8 \times 8$ Block 
In a reconstructed subimage, there are three types of errors involved: truncation error (some transform coefficients have been set to zero), quantization error, and transmission error. In a broad sense, the truncation can be viewed as a part of the quantization. That is, these truncated coefficients are quantized to zero. The transmission error in terms of bit reversal will affect the whole reconstructed subimage. This is because, in the inverse transform (such as the inverse MPDFRFT), each transform coefficient makes a contribution. In reconstructing the original image all the sub images are organized to form the whole image. Therefore the independent processing of individual sub images causes block artifacts. Though they may not severely affect the objective assessment of reconstructed image quality, block artifacts can be annoying, especially in low bit rate image coding. The selection of sub image size is important issues for implementing transform coding. In general, a large size will remove more interpixel redundancy. But it has been observed from literature that the pixel correlation becomes insignificant when the distance of pixels exceeds. On the other hand, a large size is not suitable for adaptation to local statistics, while adaptation is required in handling nonstationary images. A large size also makes the effect of a transmission error spread more widely. For these reasons, sub image size should not be large.

\section{Performance Evaluation Parameters}

Image compression using transform coding yields extremely good compression, with controllable degradation of image quality. By adjusting the cutoff of the transform coefficients, a compromise can be made between image quality and compression factor using $\mathrm{Q}$ factor. Various important image compression quality measuring parameters are Peak Signal to Noise Ratio (PSNR), Mean Square Error (MSE), Transform Coding gain (TCG) and compression ratio (CR) etc.

\section{1) Transform Coding Gain (TCG)}

One measure of energy compaction is the transform coding gain $G_{T C}$, which is defined as the ratio between the arithmetic mean and the geometric mean of the variances of all the components in the transformed vector.

$$
\mathrm{G}_{T C}=\frac{D_{D S Q}}{D_{T C}}
$$

\section{2) Peak Signal to Noise Ratio (PSNR)}

The Peak Signal-to-Noise Ratio (PSNR) value used to measure the difference between a decoded image $R(i, j)$ and its original image $L(i, j)$ is defined as follows. In general, the larger PSNR value, the better will be decoded image quality.

$$
\begin{gathered}
\text { Mean square Error(MSE) }=\frac{1}{R S} \sum_{i=1}^{R} \sum_{j=1}^{S}[L(i, j)-R(i, j)]^{2} \\
\text { PeakSignal to Noise Ratio(PSNR) }=10 \log _{10}\left[\frac{255 \times 255}{M S E}\right] d B
\end{gathered}
$$

Where $R$ and $S$ indicates the size of the image. 


\section{3) Compression Ratio (CR)}

It is defined as the ratio of size compressed image to the size of original image and is given below:

$$
\mathrm{CR} \%=\frac{\text { Size of Compressed Image }}{\text { Size of Original Image }} \times 100
$$

\section{Simulation Results, Discussion and Comparison}

In this segment, the results are obtained for satellite image, medical error image, urban image, rural image and natural image of different sizes and variety at different quality factor of image. The relationship of quality factor is associated with compression ratio mentioned below in Table 6. From the analysis of results the comparison is done between proposed scheme and already their nearby established scheme.

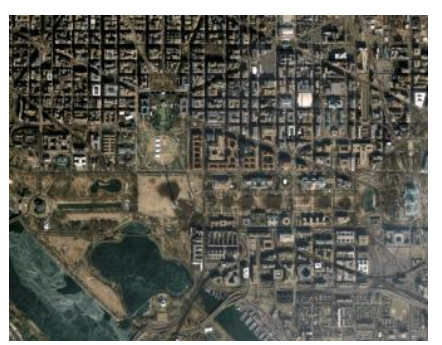

Figure 5. (a) Satellite Image (b) Decompressed at $Q=95$

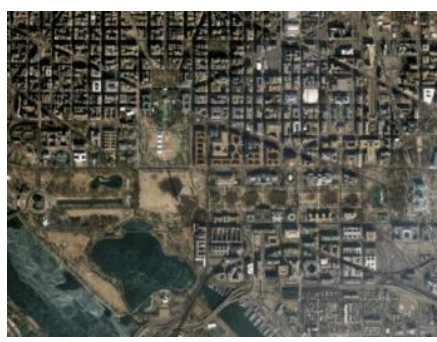

(d) At $Q=50$

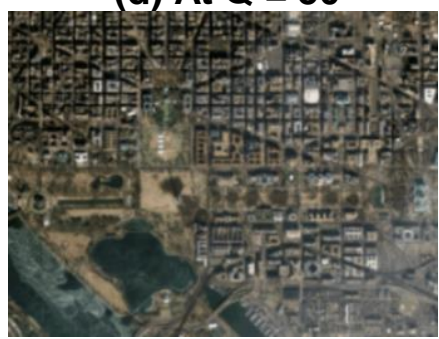

(g) At $Q=10$
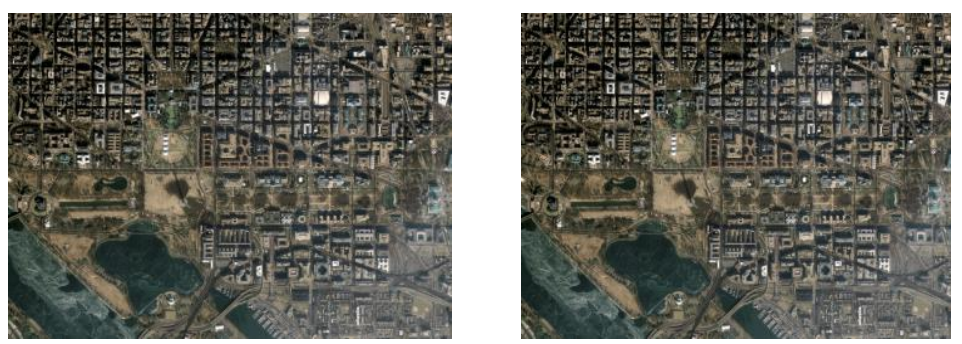

(c) At $Q=75$

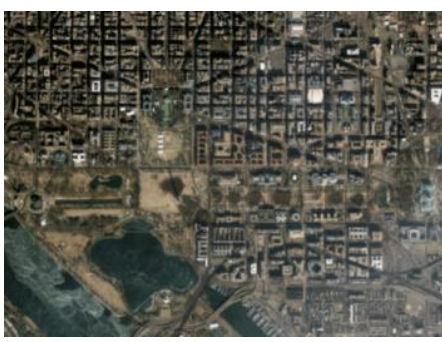

(e) At $Q=25$

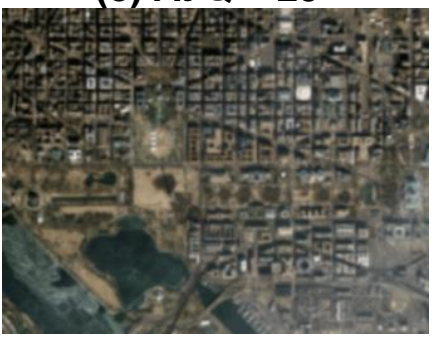

(h) At $Q=5$

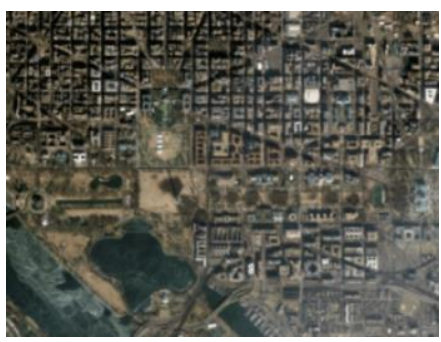

(f) At $Q=15$

The original satellite defence image is shown in Figure 5 (a) while Figure 5 (b), (c), (d), (e), (f), (g) and (h) shows the decompressed images at quality factor 95, 75, 50, 25, 15,10 and 5 respectively. The decompressed images at these quality factors recovered with their max PSNR 58.8dB, 49.2dB, 46.25dB, 45.8dB, 45.1dB, $44.7 \mathrm{~dB}$ and $44.6 \mathrm{~dB}$ respectively at the fixed fractional order parameter $\mathrm{p}=0.86$ and $\mathrm{q}=0.84$ for optimum results. The fractional parameters can be are selected in the range from 0 to 1 . 


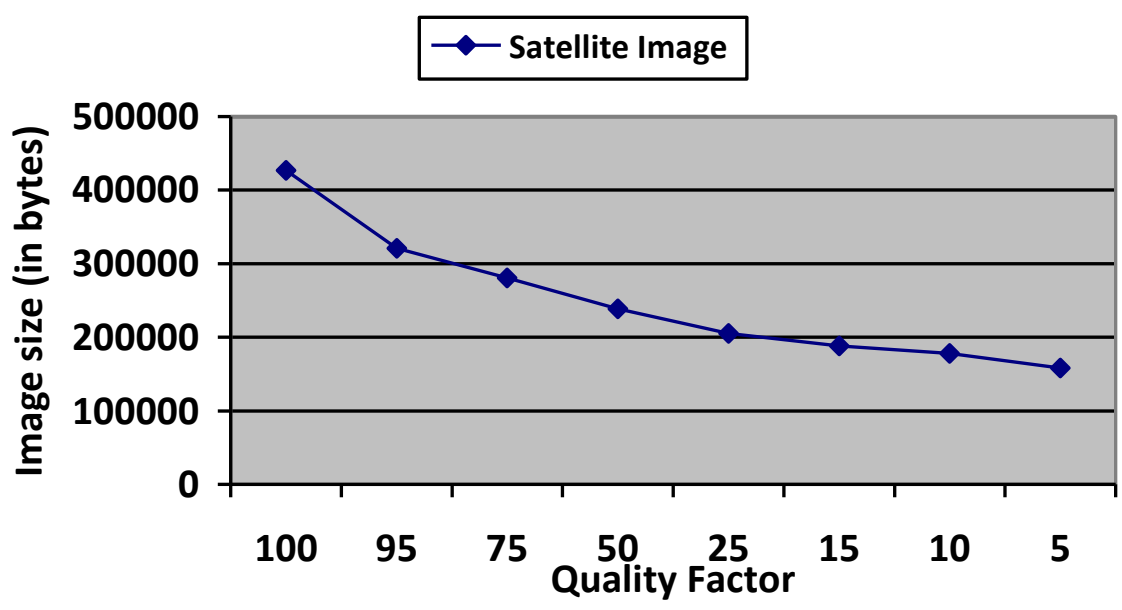

Figure 6. Variation of Image Size versus Quality Factor for Satellite Image

In Figure 6 the variation of image size versus quality factor for satellite defense image is shown it clearly depicted form the plot that the image size has been reduced up to $63 \%$ but still image quality of decompressed image is high for high resolution satellite image.
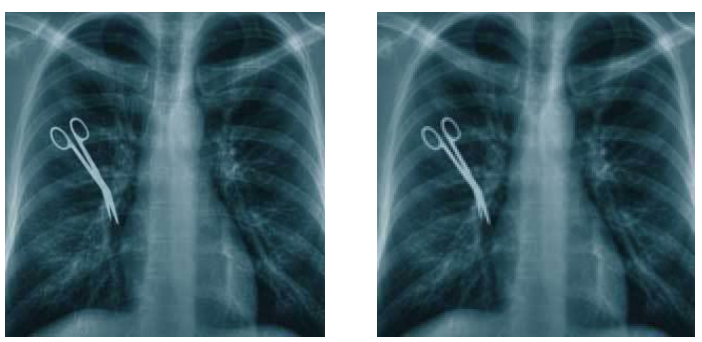

Figure 7. (a) Medical Image (b) At $Q=95$

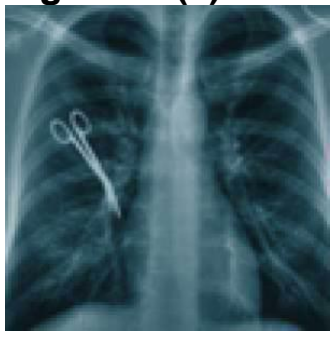

(e) At $Q=25$

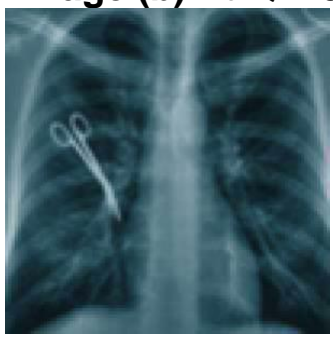

(f) At $Q=15$

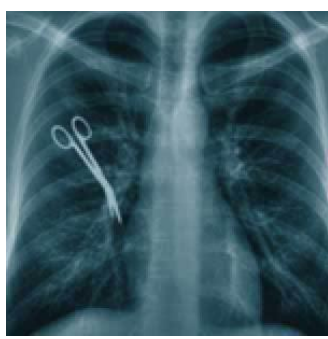

(c) At $Q=75$

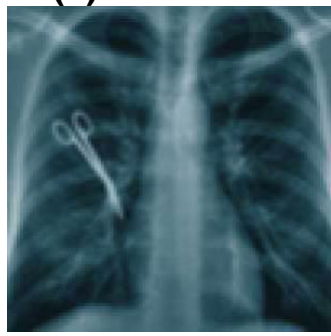

(g) At $Q=10$

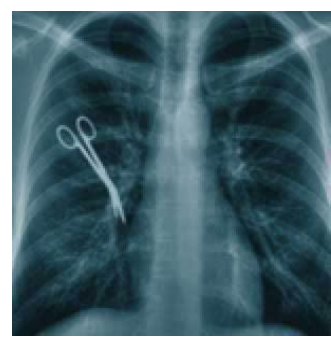

(d) At $Q=50$

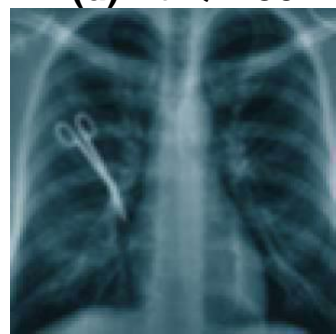

(h) At $Q=5$

The original medical error image is shown in Figure 7 (a) while in Figure 7 (b), (c), (d), (e), (f), (g) and (h) shows the decompressed images for different quality factor at $95,75,50,25,15,10$ and 5 respectively with higher medical error detection possibility apart from setting higher compression ratio. The decompressed images at these quality factors provides max PSNR of $59.1 \mathrm{~dB}, 49.6 \mathrm{~dB}, 46.2 \mathrm{~dB}, 44.3 \mathrm{~dB}, 43.9 \mathrm{~dB}, 43.3 \mathrm{~dB}$ and $44.5 \mathrm{~dB}$ respectively at the fixed fractional parameter $\mathrm{p}=0.86$ and $\mathrm{q}=0.84$. 


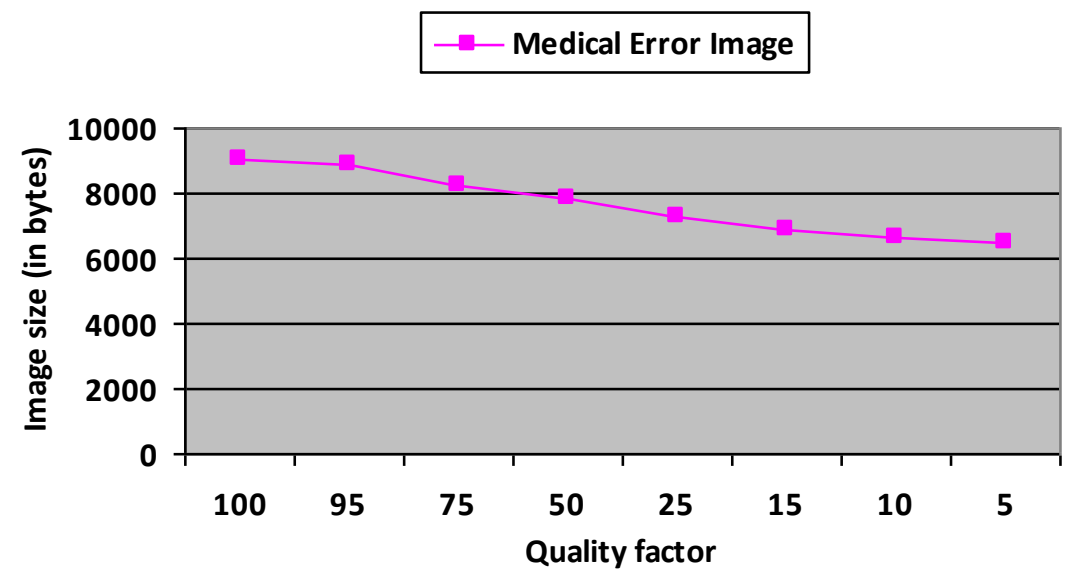

Figure 8. Variation of Image Size versus Quality Factor for Medical Error Image

The Figure 8 provides the information regarding the decompressed image size versus quality factor for medical error image and the graph shows that the image has been only has been reduced only up to $39 \%$ of its original size but still image quality of decompressed image is still has the capability of identifying any medicl error. The analysis is also done at the same fractional order parameter at $\mathrm{p}=0.86$ and $\mathrm{q}=0.84$

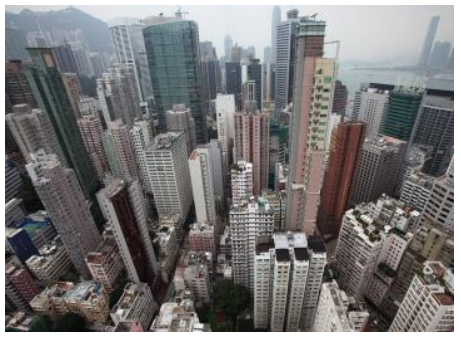

Figure 9. (a) Newyork Urban Image (b) At $Q=95$

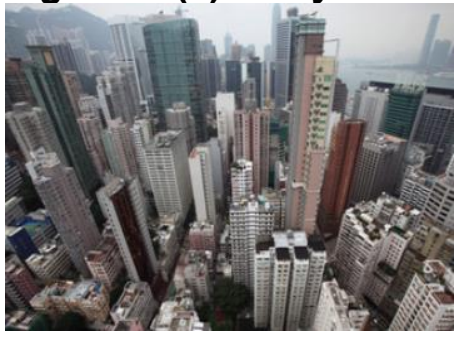

(d) At $Q=50$

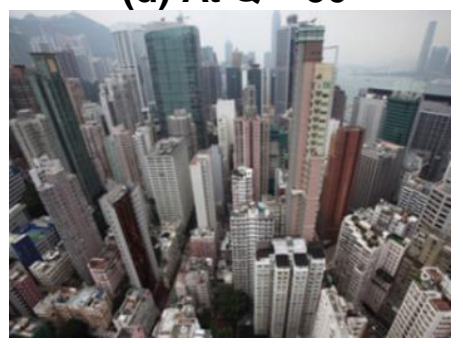

(g) At $Q=10$
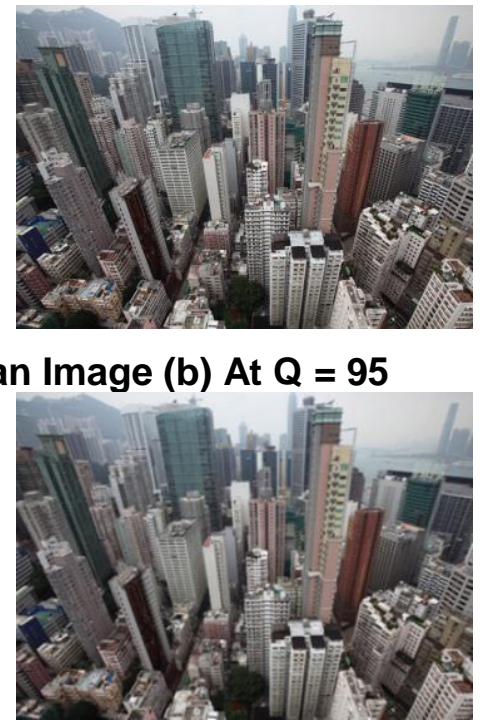

(e) At $Q=25$

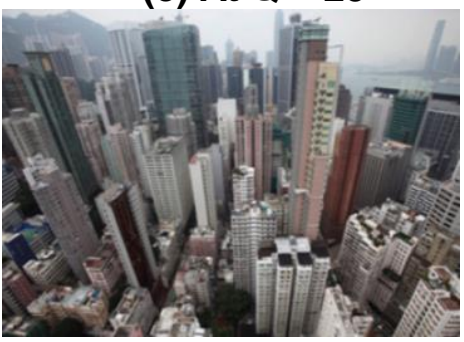

(h) At $Q=5$

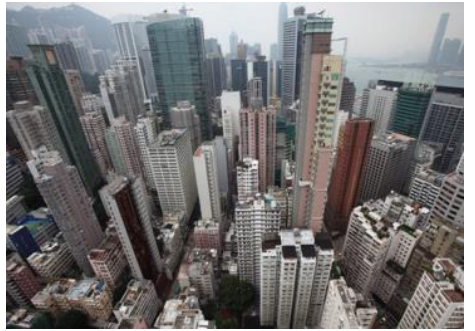

(c) At $Q=75$

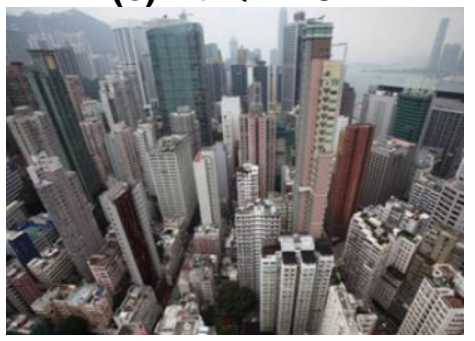

(f) At $Q=15$ 
The original urban Newyork image is shown in figure 9 (a) while in figure 9 (b), (c), (d), (e), (f), (g) and (h) shows the decompressed images for different quality factor at $95,75,50,25,15,10$ and 5 respectively with high quality reconstructed image with setting higher compression ratio. The decompressed images at these quality factors provides max PSNR of $58.8 \mathrm{~dB}, 50 \mathrm{~dB}, 48.1 \mathrm{~dB}, 46.3 \mathrm{~dB}, 45.1 \mathrm{~dB}, 44.9 \mathrm{~dB}$ and $44.5 \mathrm{~dB}$ respectively at the fixed fractional parameter $\mathrm{p}=0.86$ and $\mathrm{q}=0.84$.

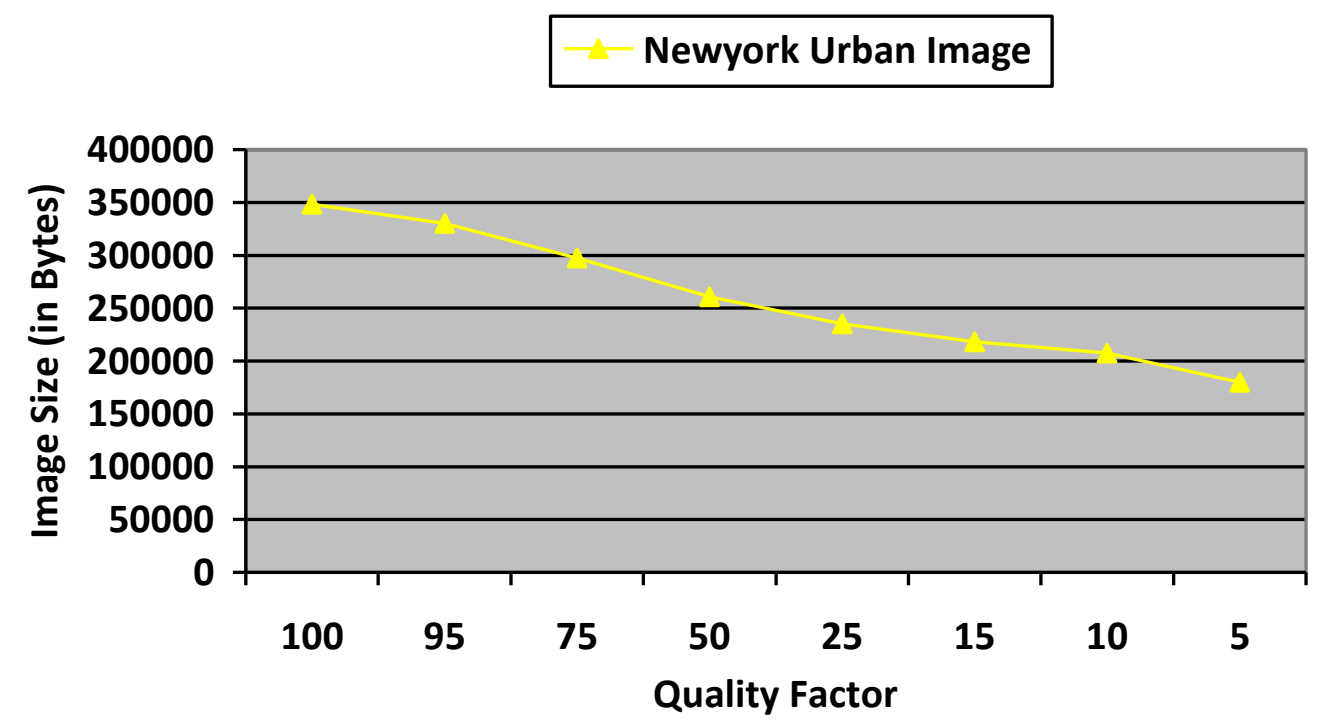

Figure 10. Variation of Image Size versus Quality Factor for Urban Image

The Figure 10 provides information between decompressed image size versus quality factor for Newyork urban image and the plot shows that the decompressed image has been reduced only up to $49 \%$ of its original size by still mainting high image quality. The fractional order parameter is selected at $\mathrm{p}=0.86$ and $\mathrm{q}=0.84$ for optimum PSNR reception.
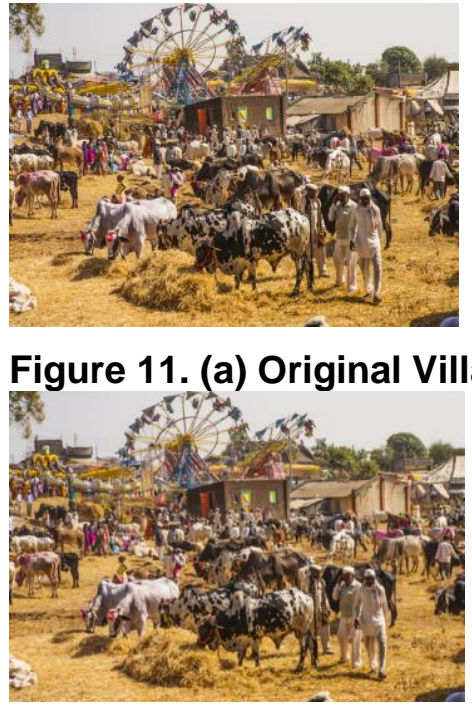

(d) At $Q=50$

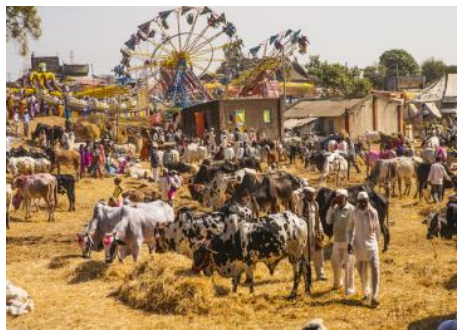

(b) At Q $=95$

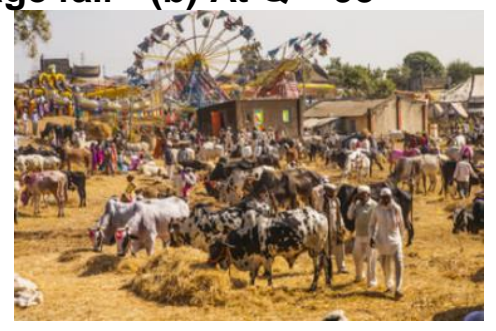

(e) At $Q=25$

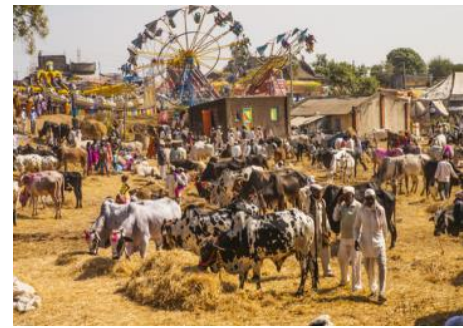

(c) At $Q=75$

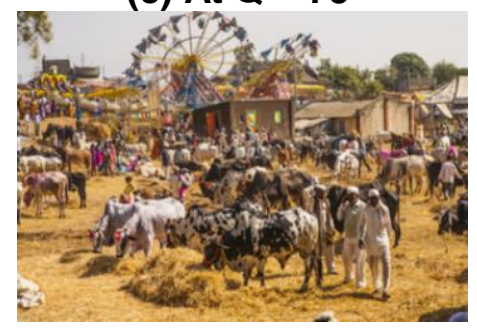

(f) At $Q=15$ 


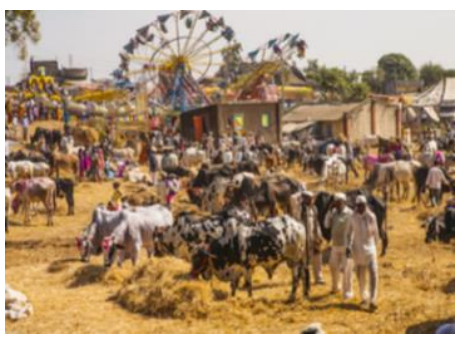

(g) At $Q=10$

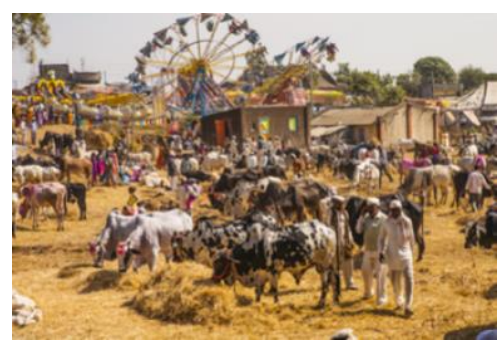

(h) At $Q=5$

The Rural village fair image of size is shown in Figure 11. (a) While Figure 11. (b), (c), (d), (e), (f), (g) and (h) shows the max PSNR for decompressed images 55.2dB, 45.1dB, $44.4 \mathrm{~dB}, 42.6 \mathrm{~dB}, 42.1 \mathrm{~dB}, 41.9 \mathrm{~dB}$ and $41.7 \mathrm{~dB}$ for different quality factor at $95,75,50,25,15$, 10 and 5 respectively. The analysis is done at fractional order parameter $p=0.86$ and $q=0.84$ for achieving max PSNR varying from 0 to 1 .

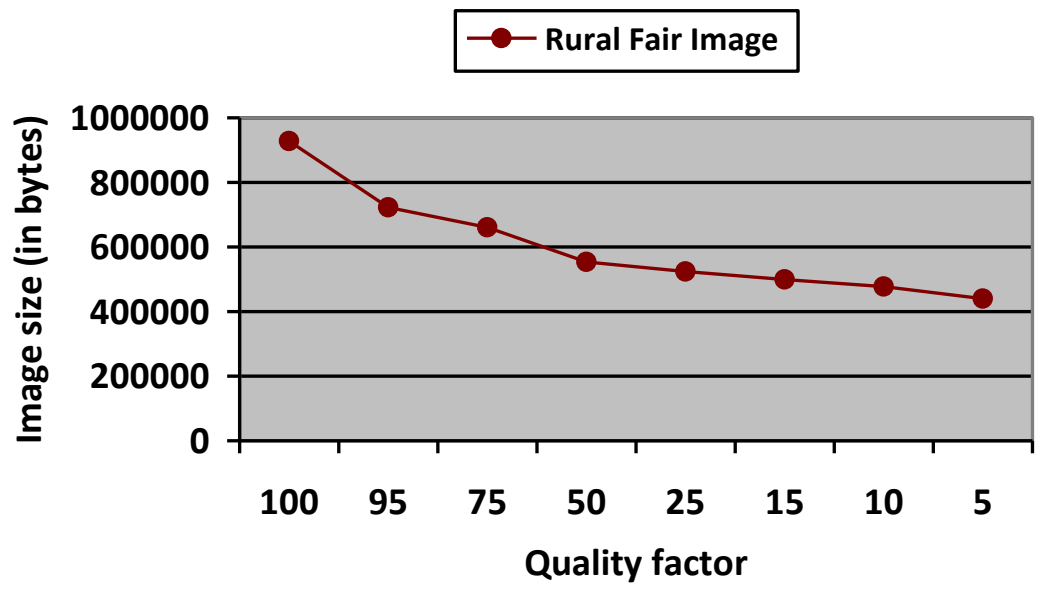

Figure 12. Variation of Image Size versus Quality Factor for Rural Village Fair

Similarly here also the Figure 12 shows the variation of decompressed image size versus quality factor for Rural Village Fair Image. This plot shows that the decompressed image has been reduced only up to $53 \%$ of its original size by still mainting high degree of image quality.

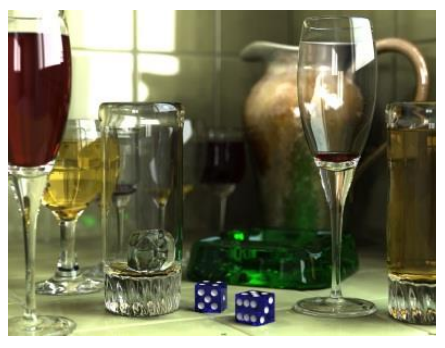

Figure 13. (a) Glass Set Image

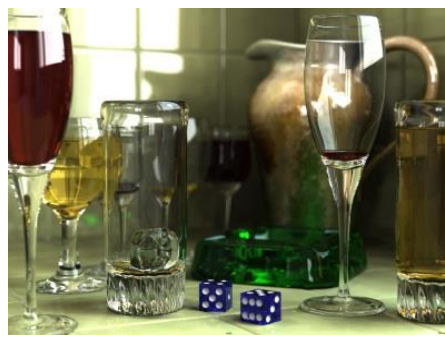

(b) At $Q=95$

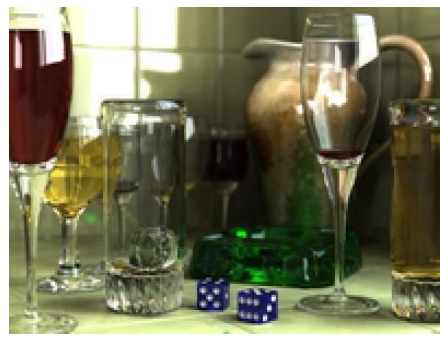

(c) At $Q=75$ 


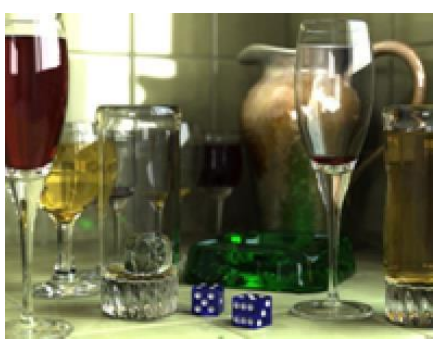

(d) At $Q=50$

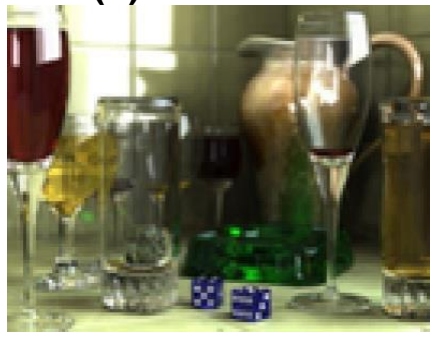

(g) At $Q=10$

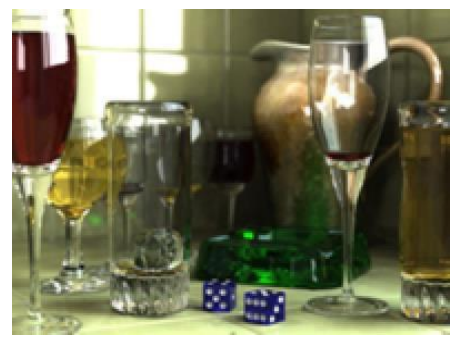

(e) At $Q=25$

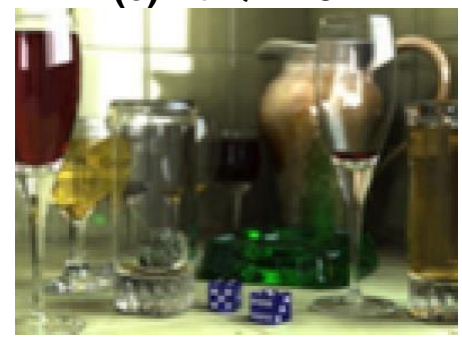

(h) At $Q=5$

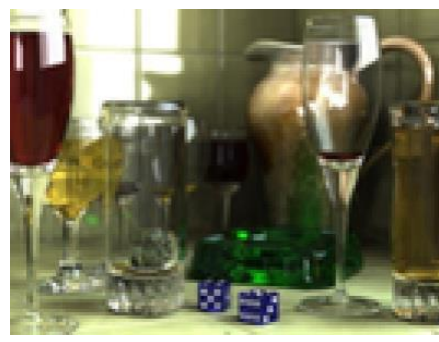

(f) At $Q=15$

The original natural glass set image is shown in Figure 13. (a) While Figure 13. (b), (c), (d), (e), (f), (g) and (h) shows the decompressed images for quality factor at 95, 75, 50, 25, 15,10 and 5 respectively. The decompressed images at these quality factors provides max PSNR $60.2 \mathrm{~dB}, 54.1 \mathrm{~dB}, 49.4 \mathrm{~dB}, 46.7 \mathrm{~dB}, 45.2 \mathrm{~dB}, 44.9 \mathrm{~dB}$ and $44.7 \mathrm{~dB}$ respectively at the fixed fractional order parameter $\mathrm{p}=0.86$ and $\mathrm{q}=0.84$. The fraction parameters are selected because at these parameters values the PSNR achieved maximum by varying $\mathrm{p}$ and $\mathrm{q}$ from 0 to 1 .

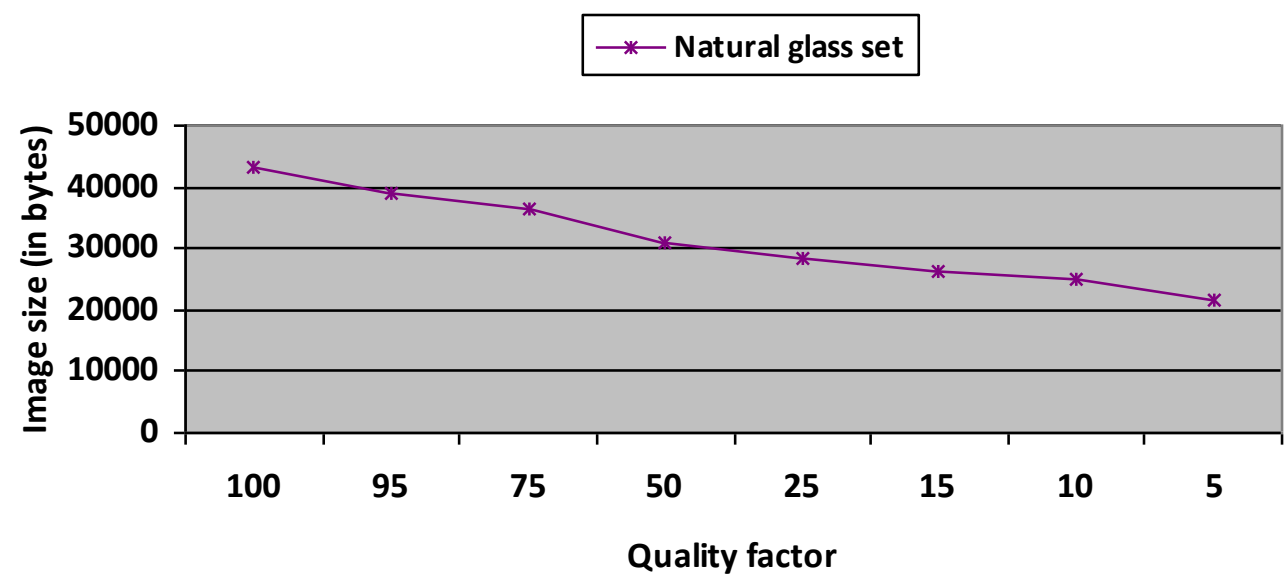

Figure 14. Variation of Image Size versus Quality Factor for Natural Glass Set

Here in Figure 14 the variation of decompressed image size versus quality factor for Natural glass set. This plot shows that the decompressed image has been reduced only approximately upto $50 \%$ of its original size by still mainting high degree of image quality. 


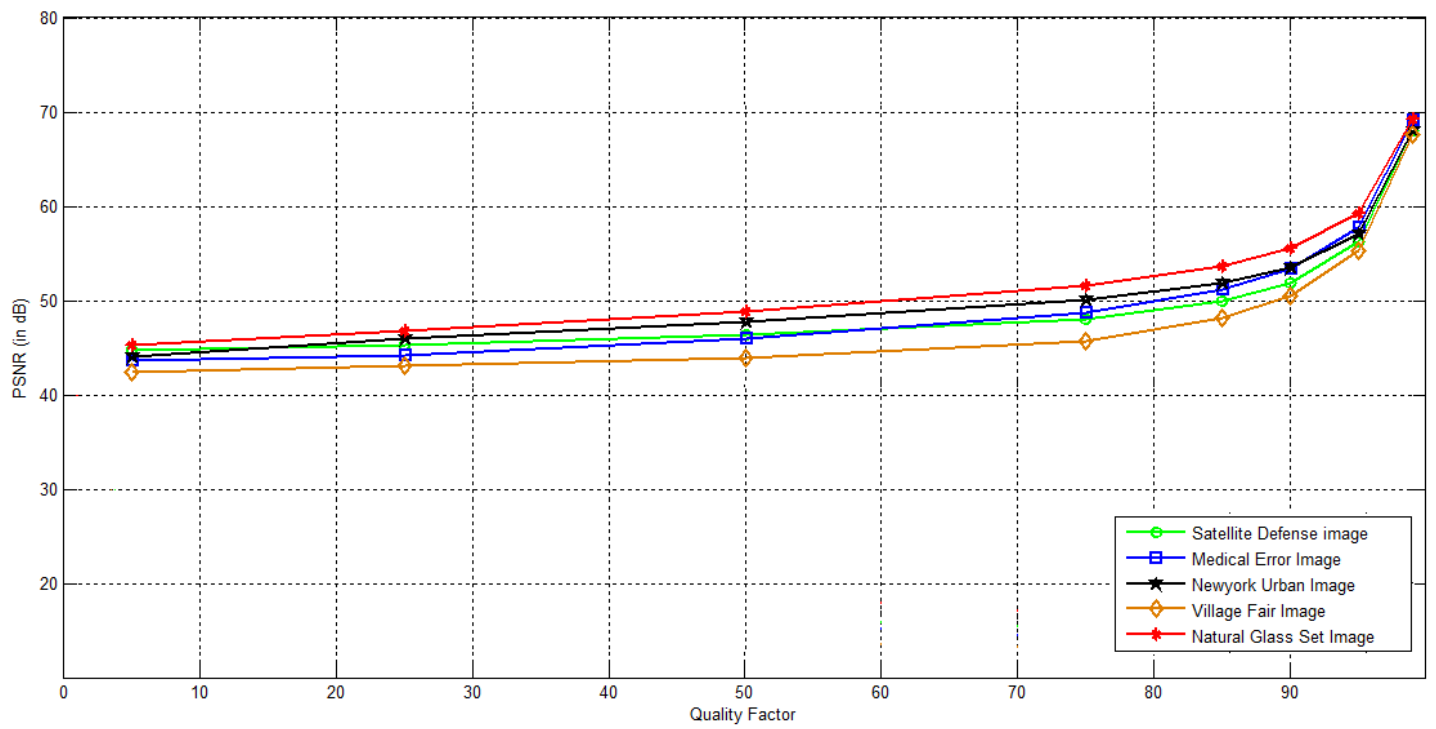

Figure 15. PSNR with Quality Factor at Fractional Order $p=0.86$ and $q=0.84$

The above Figure 15 represents the combined variation of each image PSNR versus quality factor at the fractional order $\mathrm{p}=0.86$ and $\mathrm{q}=0.84$. The graph shows maximum PSNR for Natural glass set and worst PSNR for shows for village fair image $60.2 \mathrm{~dB}$ and $41.7 \mathrm{~dB}$ respectively.

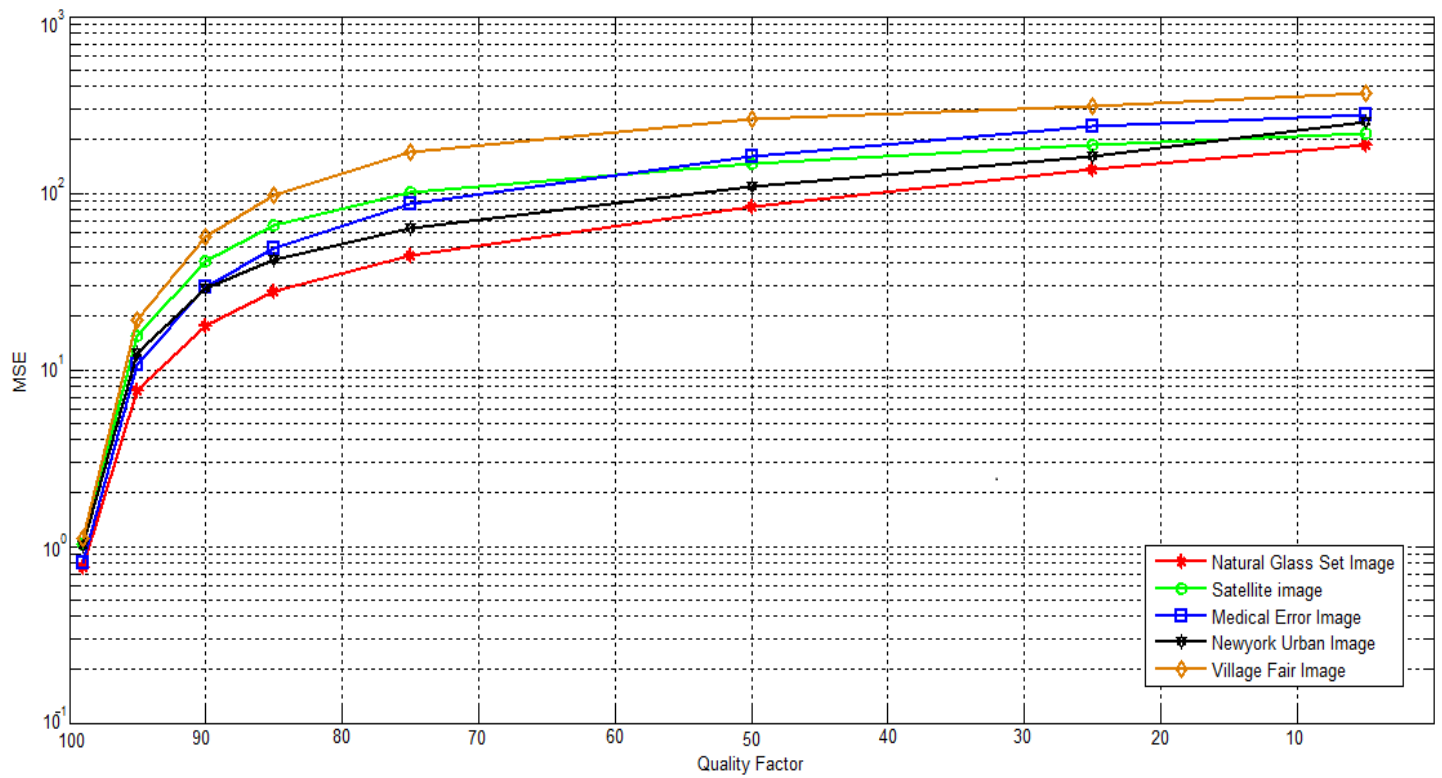

Figure 16. MSE versus Quality Factor at Fraction Order $p=0.86$ and $q=0.84$

The Figure 16 basically reprsents the dual curve of figure 16 . because the more PSNR will produce minimum MSE so here also the graph shows min. MSE for Natural glass set while worst or max MSE for village fair image are around $6 \mathrm{~dB}$ and $20 \mathrm{~dB}$ respectively. 
Table 4. Mean Square Error for Different Images at $p=0.86$ and $q=0.84$

\begin{tabular}{|c|c|c|c|c|c|}
\hline \multirow{yynnn}{*}{ Image $\rightarrow$} & $\begin{array}{c}\text { Natural Glass } \\
\text { Set Image } \\
300 \times 394 \\
\text { (Max PSNR) }\end{array}$ & $\begin{array}{c}\text { Medical Error } \\
\text { Image } \\
302 \times 302 \\
\text { At } \mathrm{M} \downarrow\end{array}$ & $\begin{array}{c}\text { Newyork } \\
\text { Urban Image } \\
974 \times 696 \\
(\text { Max PSNR) }\end{array}$ & $\begin{array}{c}\text { Satellite } \\
\text { Image } \\
916 \times 693 \\
(\text { Max PSNR) }\end{array}$ & $\begin{array}{c}\text { Rural Image } \\
683 \times 1024 \\
(\text { Max PSNR) }\end{array}$ \\
\cline { 1 - 5 } 95 & $60.2 \mathrm{~dB}$ & $59.1 \mathrm{~dB}$ & $58.8 \mathrm{~dB}$ & $58.8 \mathrm{~dB}$ & $55.2 \mathrm{~dB}$ \\
\hline 75 & $54.1 \mathrm{~dB}$ & $49.6 \mathrm{~dB}$ & $50.0 \mathrm{~dB}$ & $49.2 \mathrm{~dB}$ & $45.1 \mathrm{~dB}$ \\
\hline 50 & $49.4 \mathrm{~dB}$ & $46.2 \mathrm{~dB}$ & $48.1 \mathrm{~dB}$ & $46.25 \mathrm{~dB}$ & $44.4 \mathrm{~dB}$ \\
\hline 25 & $46.7 \mathrm{~dB}$ & $44.3 \mathrm{~dB}$ & $46.3 \mathrm{~dB}$ & $45.8 \mathrm{~dB}$ & $42.6 \mathrm{~dB}$ \\
\hline 15 & $45.2 \mathrm{~dB}$ & $43.9 \mathrm{~dB}$ & $45.1 \mathrm{~dB}$ & $45.1 \mathrm{~dB}$ & $42.1 \mathrm{~dB}$ \\
\hline 10 & $44.9 \mathrm{~dB}$ & $43.3 \mathrm{~dB}$ & $44.9 \mathrm{~dB}$ & $44.7 \mathrm{~dB}$ & $41.9 \mathrm{~dB}$ \\
\hline 5 & $44.7 \mathrm{~dB}$ & $44.5 \mathrm{~dB}$ & $44.5 \mathrm{~dB}$ & $44.6 \mathrm{~dB}$ & $41.7 \mathrm{~dB}$ \\
\hline
\end{tabular}

The Table 5 shows the response of proposed scheme towards various images like natural glass set image, medical, urban, satellite and rural images of different sizes at different $\mathrm{q}$ factorrs. Table 6 also reprsent the size of original image and decompressed image with its percentage of reduction in size with image quality in tolerable range.

Table 5. Original Image Size with Decompressed Image

\begin{tabular}{|c|c|c|c|}
\hline Type of Image & $\begin{array}{c}\text { Original Image } \\
\text { Size }\end{array}$ & $\begin{array}{c}\text { Min. } \\
\text { Decompressed } \\
\text { Image Size }\end{array}$ & $\begin{array}{c}\text { Reconstructed Image } \\
\text { Size Reduction }\end{array}$ \\
\hline Satellite Defense Image & 426810 bytes & 158068 bytes & $63 \%$ \\
\hline Medical Error Image & 9034 bytes & 6451 bytes & $39 \%$ \\
\hline Rural Fair Image & 927201 bytes & 440522 bytes & $53 \%$ \\
\hline Newyork Urban Image & 348185 bytes & 180078 bytes & $49 \%$ \\
\hline Natural Glass Set Image & 43139 bytes & 21808 bytes & $50 \%$ \\
\hline
\end{tabular}

The Table 6 shows the important relationship between the quality factor and compression ratio so by setting quality factor proportionally compression ration can be achieved.

\section{Table 6. Relationship between Quality Factor and Compression Ratio for JPEG}

\begin{tabular}{|c|c|}
\hline Quality Factor & Compression Ratio (CR) \\
\hline 100 & $1: 1$ \\
\hline 95 & $2: 1$ \\
\hline 75 & $15: 1$ \\
\hline 50 & $23: 1$ \\
\hline 25 & $46: 1$ \\
\hline 1 & $144: 1$ \\
\hline
\end{tabular}

The Table 7 concludes the previous performance Table 4 along with the comparison of already existed schemes. The proposed scheme is compared with the schemes mentioned in [17-19] interms of PSNR, computation complexity and transform coding gain. 
Table 7. Comparison of Proposed Scheme with Established Schemes

\begin{tabular}{|c|c|c|c|c|c|}
\hline Schemes & Type of Image & $\begin{array}{l}\text { Maximum } \\
\text { PSNR }\end{array}$ & $\begin{array}{l}\text { Minimum } \\
\text { MSE }\end{array}$ & $\begin{array}{l}\text { Computation } \\
\text { Complexity }\end{array}$ & $\begin{array}{l}\text { Transform } \\
\text { Coding } \\
\text { Gain }\end{array}$ \\
\hline \multirow{5}{*}{$\begin{array}{l}\text { Proposed } \\
\text { Scheme }\end{array}$} & $\begin{array}{l}\text { Natural Glass } \\
\text { Set image }\end{array}$ & $69.8 \mathrm{~dB}$ & $6 \mathrm{~dB}$ & $O\left(N^{2}\right)$ & High \\
\hline & $\begin{array}{l}\text { Medical Error } \\
\text { Image }\end{array}$ & $69.6 \mathrm{~dB}$ & $11 \mathrm{~dB}$ & $O\left(N^{2}\right)$ & High \\
\hline & $\begin{array}{c}\text { Newyork Urban } \\
\text { Image }\end{array}$ & $69.4 \mathrm{~dB}$ & $12 \mathrm{~dB}$ & $O\left(N^{2}\right)$ & High \\
\hline & $\begin{array}{c}\text { Satellite } \\
\text { Defences Image }\end{array}$ & $69.3 \mathrm{~dB}$ & $16 \mathrm{~dB}$ & $O\left(N^{2}\right)$ & High \\
\hline & $\begin{array}{l}\text { Rural Village } \\
\text { Fair Image } \\
\end{array}$ & $68.8 \mathrm{~dB}$ & $20 \mathrm{~dB}$ & $O\left(N^{2}\right)$ & High \\
\hline \multirow{2}{*}{$\begin{array}{l}\text { Kulbir Singh } \\
\text { et.al. [17] }\end{array}$} & Leena Image & $27.5 \mathrm{~dB}$ & --- & \multirow{2}{*}{ Low } & \multirow{2}{*}{ Low } \\
\hline & Barbara Image & $27.8 \mathrm{~dB}$ & --- & & \\
\hline $\begin{array}{l}\text { K. Nagmani and } \\
\text { A.G. Anant [18] }\end{array}$ & Satellite Image & $29.0 \mathrm{~dB}$ & & $\begin{array}{c}\text { Lower than } \\
O\left(N^{2}\right)\end{array}$ & Medium \\
\hline $\begin{array}{c}\text { Rajinder Kumar } \\
\text { et.al.[19] }\end{array}$ & Satellite Image & $41.0 \mathrm{~dB}$ & & $O\left(N^{2}\right)$ & High \\
\hline
\end{tabular}

\section{Conclusion}

In this article, a new image compression scheme based on multiple parameter discrete fractional Fourier transform is proposed. This scheme shows a max. PSNR for Natural glass set image $60.2 \mathrm{~dB}$ while min. PSNR for village fair is $41.7 \mathrm{~dB}$. Table 5 shows that the satellite defence image shows the maximum compressed image upto $60 \%$ with significant image quality with respect to original image size and for medical error image shows large decompressed size with $39 \%$ but medical error can be detected for smaller quality factor also and can be reconstructed with little larger size but can be useful for more medical applications. The proposed scheme also shows high PSNR, $O\left(N^{2}\right)$ computational complexity and higher coding efficiency than FRCT, FRFT and DFRFT based image compression schemes.

\section{Acknowledgements}

The authors thankfully acknowledge all the authorities of Jaypee University of Engineering \& Technology, Guna (M.P.), PIN-473226, India.

\section{References}

[1] Y. Q. Shi and Huifang Sun, "Image and video compression for multimedia engineering: Fundamentals, algorithms and standards", CRC Press Taylor \& Francis Group, New York, (2000).

[2] K. R. Rao and P. C. Yip, "The transform and data compression handbook", CRC Press Taylor \& Francis Group, New York, (2000).

[3] H. M. Ozaktas, Z. Zalevsky and M. A. Kutay, "The Fractional Fourier Transform with Applications in Optics and Signal Processing", John Wiley, New York, USA, (2001).

[4] V. Namias, "The fractional order Fourier transform and its application to quantum mechanics", IMA Journal of Applied Mathematics, vol. 25, no. 3, (1980), pp. 241-265. 
[5] B. Santhanam and J. H. McClellan, "The discrete rotational Fourier transform”, IEEE Trans. Signal Process., vol. 44, no. 4, (1996), pp. 994-998.

[6] B. W. Dickinson and K. Steiglitz, "Eigenvalues and eigenvectors of the discrete Fourier transform", IEEE Trans. Acoust. Speech Signal Process, vol. 30, no. 1, (1982), pp. 25-31.

[7] C. Candan, M. A. Kutay and H. M. Ozaktas, "The discrete fractional Fourier transform", IEEE Trans. on Signal Process, vol. 48, no. 5, (2000), pp. 1329-1337.

[8] C. Candan, "On higher order approximations for Hermite-Gaussian functions and discrete fractional Fourier transforms", IEEE Signal Process. Lett., vol. 14, no. 10, (2007), pp. 699-702.

[9] S. C. Pei and M. H. Yeh, "Improved discrete fractional Fourier transform," Opt. Lett., vol. 22, no. 14, (1997), pp. 1047-1049.

[10] M. T. Hanna, N. P. A. Seif and W. A. E. M. Ahmed, "Hermite- Gaussian-Like eigenvectors of the discrete Fourier transform matrix based on the singular value decomposition of its orthogonal projection matrices," IEEE Trans. on Circuits and Systems, vol. 51, no. 11, (2004) pp. 2245-2254.

[11] M. T. Hanna, "Direct batch evaluation of optimal orthonormal eigenvectors of the DFT matrix", IEEE Trans. Signal Process., vol. 56, no. 5, (2008), pp. 2138-2143.

[12] M. T. Hanna, N. P. A. Seif and W. A. E. M. Ahmed, "Hermite- Gaussian-Like eigenvectors of the discrete Fourier transform matrix based on the direct utilization of the orthogonal projection matrices on its eigenspaces”, IEEE Trans. Signal Process., vol. 54, no. 7, (2006), pp. 2815-2819.

[13] L. B. Almeida, "The fractional Fourier transform and time-frequency representations", IEEE Trans. Signal Process., vol. 42, no. 11, (1994), pp. 3084-3091.

[14] A. Sahlin, H. M. Ozaktas and D. Mendlovic, "Optical implementation of the two-dimensional fractional Fourier transform with different orders in the two dimensions" Elsevier Optics Communication, vol. 120, issue 3-4 (1995), pp. 134-138.

[15] S. C. Pei, M. H. Yeh and C. C. Tseng, "Discrete fractional Fourier transform based on orthogonal projections," IEEE Trans. Signal Processing, vol. 47, (1999) May, pp. 1335-1348.

[16] W. H. Chen and W. K. Pratt, "Scene adaptive coder", IEEE Transaction on Communication, COM-32, no.3, (1984), pp. 225-232.

[17] K. Singh, N. Singh, P. Kaur and R. Saxena, "Image Compression By Using Fractional Transforms" International Conference on Advances in Recent Technologies in Communication and Computing (ARTCOM), Kottayam, Kerala, India, (2009), pp. 411-413.

[18] K. Nagmani and A. G. Anant, "Image Compression Techniques for High Resolution Satellite Imageries using Classical Lifting Scheme", International Journal of Computer Applications, vol. 15, no. 3, (2011), pp. 25-28.

[19] R. Kumar, K. Singh and R. Khanna, "Satellite Image Compression using Fractional Fourier Transform" International Journal of Computer Applications (0975 - 8887), vol. 50, no. 3, (2012), pp. 20-25.

\section{Authors}

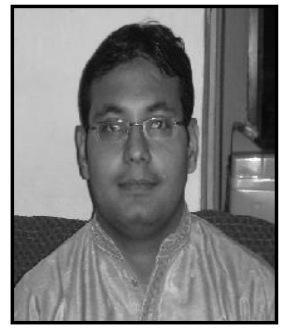

Deepak Sharma, obtained his M. Tech. degree (Microwave Engineering) from Madhav Institute of Technology and Science in 2006. Currently working as an Assistant Professor in Jaypee University of Engineering and Technology (JUET), Guna before joining JUET, he worked as a Lecturer in Electronics Department, MITS, Gwalior (M.P). Currently, pursuing Ph.D. degree from Jaypee University of Engineering and Technology, Guna. His Research areas include Antenna Theory, Radar System, Signal processing, Image processing and Integral Transforms.

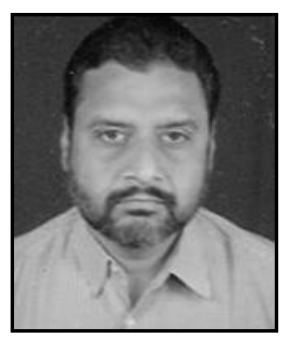

Rajiv Saxena, born at Gwalior in Madhya Pradesh in 1961, obtained B.E. (Electronics \& Telecommunication Engineering) in the year 1982 from Jabalpur University, Jabalpur. Subsequently, Dr. Saxena joined the Reliance Industries, Ahmedabad, as Graduate Trainee. In 1984, Dr. Saxena joined Madhav Institute of Technology $\&$ Science, Gwalior as Lecturer in Electronics Engineering. He 
obtained his M.E. (Digital Techniques \& Data Processing) from Jiwaji University, Gwalior in 1990. The Ph. D. degree was conferred on him in 1996-97 in Electronics \& Computer Engineering from IIT, Roorkee (erstwhile UOR, Roorkee). Dr. Saxena was former head and professor at Jaypee University, Guna (M.P.) for seven year. Currently, Dr. Saxena is Director at Jaypee University, Anupshahar (U.P.).

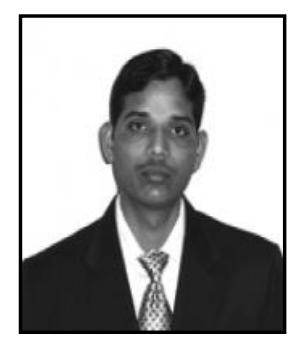

Narendra Singh, born at Orai in Uttar Pradesh in 1977, obtained B. Tech. (Electronics \& Instrumentation Engineering) in the year 2001 from Bundelkhand University, Jhansi, M. Tech. in Digital Systems in the year 2004 from M.N.N.I.T. Allahabad (Deemed University) and obtained Ph.D. from Jaypee University of Engineering \& Technology, Guna, (M.P.) in the year 2012. Narendra Singh joined JIET, Guna, as lecturer in Electronics and Communication Engineering in 2004. Currently he is assistant professor (senior grade) in ECE department at JUET, Guna. 